\title{
Influence of lanthanum doping on the morphotropic phase boundary of lead zirconate titanate
}

\author{
Manuel Hinterstein, ${ }^{1, a)}$ Kristin A. Schoenau, ${ }^{1}$ Jens Kling, ${ }^{1}$ Hartmut Fuess, ${ }^{1}$ Michael Knapp, ${ }^{2}$ \\ Hans Kungl, ${ }^{3}$ and Michael J. Hoffmann ${ }^{3}$ \\ ${ }_{1}^{1}$ Institute of Materials Science, TU Darmstadt, Petersenstr. 23, D-64287 Darmstadt, Germany \\ ${ }^{2}$ CELLS, P.O. Box 68, 08193 Barcelona, Spain \\ ${ }^{3}$ Institute of Ceramics in Mechanical Engineering, University of Karlsruhe, 76131 Karlsruhe, Germany
}

(Received 11 January 2010; accepted 2 May 2010; published online 26 July 2010)

\begin{abstract}
A phase diagram for morphotropic $\left(\mathrm{Pb}_{0.985} \mathrm{La}_{0.01}\right)\left(\mathrm{Zr}_{1-x} \mathrm{Ti}_{x}\right) \mathrm{O}_{3}$ is proposed based on a combination of X-ray and neutron powder diffraction experiments and complemented by transmission electron microscopy. Dependent on composition three regions are characterized. The stability range of tetragonal microdomains for high Ti contents, the stability range of rhombohedral microdomains for low Ti contents, and an intermediate stability range of nanodomains. All three regions exhibit a corresponding low temperature configuration. Temperature dependent diffraction revealed that lanthanum doping reduces the sensitivity of the structure to changes in temperature and composition. A continuous transition from pseudorhombohedral to tetragonal symmetry with an intermediate two-phase region at the morphotropic phase boundary is observed. A similar transition of low temperature superstructure phases from pseudorhombohedral to pseudotetragonal with an intermediate monoclinic phase governed by a continuous change in the oxygen octahedral tilt system from $a^{-} a^{-} a^{-}$over $a^{-} a^{-} c^{-}$to $a^{0} a^{0} c^{-}$is identified. (C) 2010 American Institute of Physics.
\end{abstract} [doi:10.1063/1.3437399]

\section{INTRODUCTION}

Ferroelectric ceramics are used as piezoelectric components and optical devices or in microelectronics. One of the most frequently applied materials is the solid solution $\mathrm{PbZr}_{1-x} \mathrm{Ti}_{x} \mathrm{O}_{3}$ (PZT). Highest macroscopic piezoelectric properties have been assigned to a new structural modification ${ }^{1}$ and are observed at the so called morphotropic phase boundary (MPB) $)^{2}$ near $x=0.5$.

The Ti rich side of the PZT phase diagram is generally accepted to have tetragonal structure described with space group P $4 \mathrm{~mm}\left(C_{4 v}^{1}\right)$. On the rhombohedral side two ferroelectric phases could be identified as $R 3 m\left(C_{3 v}^{6}\right)$ for high temperatures and $R 3 c\left(C_{3 v}^{5}\right)$ for low temperatures. ${ }^{3}$

The $R 3 c$ phase is described by cation displacement along $[111]_{c}$ and oxygen octahedral tilts of the type $a^{-} a^{-} a^{-}$. The subscript ' $c$ ' denotes the pseudocubic perovskite unit cell. Noheda et al. ${ }^{1}$ proposed an intermediate monoclinic phase with space group $\mathrm{Cm}\left(C_{s}^{3}\right)$ as a bridging phase between the tetragonal and the rhombohedral regions. Additionally Hatch et al. $^{5}$ and Pandey and Ragini ${ }^{6}$ described the low temperature phase at the MPB with space group $C c\left(C_{5}^{4}\right)$ exhibiting octahedral tilting of the type $a^{-} a^{-} c^{-}$. From first principle calculations Kornev et al. ${ }^{7}$ postulated a tetragonal low temperature phase with space group $I 4 \mathrm{~cm}\left(C_{4 v}^{10}\right)$ and octahedral tilting of the type $a^{0} a^{0} c^{-}$.

The discovery of a monoclinic phase was in agreement with the work of Corker et al., ${ }^{8}$ who proposed a local monoclinic structure for rhombohedral PZT. With the refinement of anisotropic displacement ellipsoids they showed, that disordered $\mathrm{Pb}$ cation displacement could still show a rhombo-

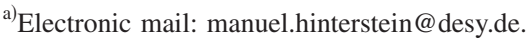

hedral structure on average. This idea was extended by Glazer et $a l .{ }^{9}$ to the tetragonal side across the MPB. They suggested that PZT follows a composition dependent progression from short range to long range and back to short range order by crossing the MPB. They concluded that all of the three proposed regions of different structures of PZT, the tetragonal, the monoclinic, and the rhombohedral region could actually be described in terms of monoclinic order at a local level "provided one considers the structures on a suitably small length scale."

Despite extensive studies the structural properties of the MPB in these ferroelectric materials are still under discussion. Derived from Rietveld refinement of diffraction data using three-dimensional (3D) periodic structural models, some groups (Noheda et al. ${ }^{10}$ and Pandey et al. ${ }^{11}$ ) assume the structure to exist as a single monoclinic $\mathrm{Cm}$ phase in the area of the MPB, as R-values of the fit are lowest.

However, ferroelectric materials with low domain wall energy tend to exhibit a complex domain configuration: transmission electron microscopy (TEM) experiments of materials with MPB compositions show a complex system of micro- and nanodomains. ${ }^{12-16}$ Schmitt et al. ${ }^{17}$ showed that the size of nanodomains increases at the MPB of PZT, however, still containing a set of subdomains within. Schierholz et al. ${ }^{18,19}$ on the other hand discovered with convergent beam electron diffraction, that for morphotropic compositions locally monoclinic symmetry is present within single domains while in other areas nanostructures are observed.

This complex structure which is definitely not preserved in three dimensions, can strongly influence its diffraction pattern (DP). Boysen ${ }^{20}$ recently showed that a tetragonal set of twin structures of several hundred Ångström width can 
$\mathrm{a}_{\mathrm{m}}=\mathrm{b}_{\mathrm{m}} \neq \mathrm{c}_{\mathrm{m}}, \beta_{\mathrm{m}} \approx 90^{\circ} \quad$ pseudotetragonal
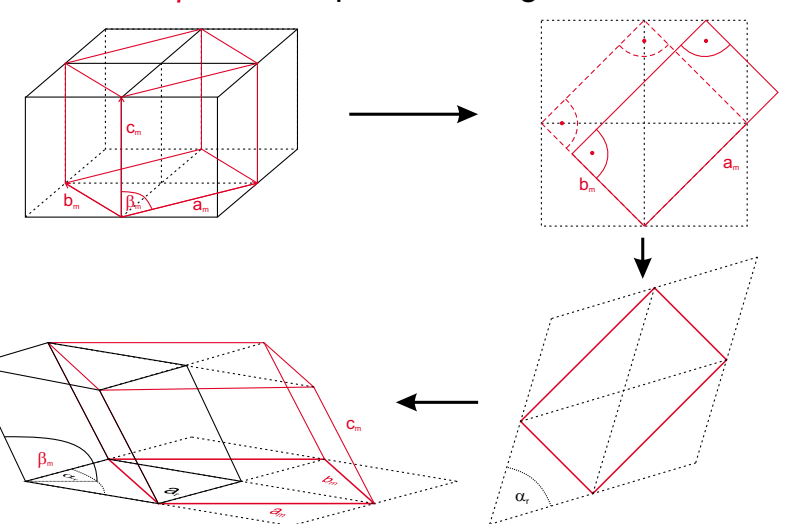

$\mathrm{a}_{\mathrm{r}} \approx \mathrm{C}_{\mathrm{m}}, \cos \beta_{\mathrm{m}} \approx-2\left(\frac{\mathrm{a}_{\mathrm{t}}}{\mathrm{a}_{\mathrm{m}}}\right) \cos \alpha_{\mathrm{r}}$

pseudorhombohedral

FIG. 1. (Color online) Transition from pseudotetragonal to pseudorhombohedral symmetry on the basis of the monoclinic model phase with space group $\mathrm{Cm}$.

severely influence its resulting DP through coherence effects, a theory also followed by various theoretical studies by Wang. ${ }^{21}$ Furthermore, besides coherence effects, strain causes anisotropic peak broadening. ${ }^{14}$

It is, therefore, not evident to determine the internal structure of a highly correlated system of domains as in PZT unambiguously by diffraction experiments, as previously claimed by Yokota et al. ${ }^{22}$ Despite the internal symmetry of the domains, the resulting DP will appear to be of lower symmetry or varied in such a way, that only a lowering of the symmetry in a Rietveld fit will result in an adequate modeling of the data. The use of anisotropic thermal displacement parameters may smooth diffraction profile mismatch. In a previous paper ${ }^{13}$ our group proposed the monoclinic $\mathrm{Cm}$ space group in order to describe the $3 \mathrm{D}$ periodicity by a Rietveld refinement of morphotropic PZT as a model system for a nanostructure configuration. ${ }^{13}$ Further examination of the material using methods other than diffraction with different length scales of analysis are required to obtain additional information on structural properties.

As the domain structure of unpoled samples at the MPB consists of a combination of tetragonal microdomains together with nanodomains or rhombohedral microdomains, ${ }^{17,18}$ a two-phase Rietveld fit needs to be applied. ${ }^{13}$ In the presence of nanodomains the crystallographic structure is averaged over several domains. The superposition of scattered intensities is approximated in the fit with $\mathrm{Cm}$ symmetry and a pseudocubic $c_{m} / a_{p c}$ ratio well above 1 results.

Furthermore, strain generated between different domains can cause anisotropic peak broadening of distinct reflections. ${ }^{14}$ A Ti content below $47 \%$ leads to rhombohedral microdomains and nanodomains gradually disappearing with $x .{ }^{12}$ The monoclinic phase may thus be interpreted as pseudorhombohedral.

Since $\mathrm{Cm}$ is a subgroup of both $P 4 \mathrm{~mm}$ and $R 3 \mathrm{~m}$ the monoclinic setup offers two parameters of interpretation. First the ratio between the lattice parameters $a_{m}$ and $b_{m}$, which results in a nonrectangular pseudocubic $\{001\}_{c}$ plane as depicted in Fig. 1 and second the relation between the resulting pseudorhombohedral angle $\alpha_{r}$ and the monoclinic angle $\beta_{m}$.

Together with the atomic positions the spontaneous polarization and its direction in the $\{1 \overline{1} 0\}_{c}$ plane gives additional information about the structural characteristics of the monoclinic phase. The spontaneous polarization $P_{s}$ can be estimated with the cell parameters and the atomic positions of the $\mathrm{Cm}$ phase by the dipole moment equation $P_{s}$ $\approx(1 / V) \sum_{i} q_{i} r_{i}$, where the summation is over the point charges $q_{i}$ at positions $r_{i}$ in a primitive cell volume $V .^{23}$

The lattice parameters for a rhombohedral phase, recalculated from the monoclinic cell to pseudocubic axes, have a $c_{m} / a_{p c}$ ratio of around 1 and additionally

$$
\cos \beta_{m} \approx-2\left(\frac{a_{r}}{a_{m}}\right) \cos \alpha_{r}
$$

where

$$
a_{r}=\frac{1}{2} \sqrt{a_{m}^{2}+b_{m}^{2}},
$$

is the pseudorhombohedral lattice parameter, $\alpha_{r}$ the pseudorhombohedral angle, $\beta_{m}$ the monoclinic angle, and $a_{m}$ and $b_{m}$ the monoclinic lattice parameters. Figure 1 describes the symmetry of the model phase $\mathrm{Cm}$. If $a_{m} \approx b_{m}\left(\alpha_{r} \approx 90^{\circ}\right)$ and $\beta_{m} \approx 90^{\circ}$ the structure can be interpreted as pseudotetragonal. For nanodomains $\beta_{m}$ is well above $90^{\circ}$. If $a_{m} \neq b_{m}$ the resulting pseudocubic $\{001\}_{c}$ planes are deformed in a rhombic way leading to a rhombohedral angle

$$
\alpha_{r}=2 \arctan \frac{b_{m}}{a_{m}} .
$$

If additionally Eq. (1) is fulfilled the structure can be described by rhombohedral microdomains. The tetragonal phase $P 4 \mathrm{~mm}$ is interpreted as tetragonal microdomains.

Thus the amount of tetragonal microdomains on one hand and nanodomains or rhombohedral microdomains on the other hand can be estimated using tetragonal $P 4 \mathrm{~mm}$ and monoclinic $\mathrm{Cm}$ phases in the Rietveld fit to monitor changes in lattice parameters and phase fractions under varying temperatures. The $c_{m} / a_{p c}$ ratio and the relation between the monoclinic angle $\beta_{m}$ and the pseudorhombohedral angle $\alpha_{r}$ [Eq. (1)] are used to distinguish between nanodomains and rhombohedral microdomains.

Curie temperature and dielectric properties of the system are modified by the incorporation of various additives. ${ }^{24} \mathrm{De}-$ pendent on atomic radii, substitution can take place on the Aor $\mathrm{B}$-site of the $\mathrm{ABO}_{3}$ perovskite structure using isovalent or aliovalent ions. For bulk PZT material, most studies focus on two types of dopants, namely, acceptors and donors, both substituting $\mathrm{A}^{2+}$ - and $\mathrm{B}^{4+}$-site ions. Higher valence dopants like $\mathrm{La}^{3+}$ and $\mathrm{Nb}^{5+}$ contribute electrons, thus act as donors and substitute the A- and B-site, respectively, ${ }^{25-28}$ creating A-site vacancies $\left(\mathrm{V}_{\mathrm{Pb}}^{\prime \prime}\right){ }^{29}$ Lower valence ions, like $\mathrm{Na}^{+}$and $\mathrm{Fe}^{3+}$, act as an acceptor dopant and may substitute the A- and $\mathrm{B}$-site, respectively, creating oxygen vacancies $\left(\mathrm{V}_{\mathrm{O}}^{*}\right)^{30,31}$

The induced lead vacancies reduce lattice and domain wall energy, therefore, facilitating domain motions. The high mobility of domain walls in donor doped ceramics leads to 
an increase in dielectric loss and this also increases the dielectric constant as well as the spontaneous polarization, resulting in more charges generated. ${ }^{32,33}$ Due to these characteristics, donor doped PZT is termed "soft." Among donor dopants in particular $\mathrm{La}^{3+}$ ions are known to promote uniform grain growth and homogeneous densification of a single phase microstructure with low porosity. ${ }^{27}$ For lanthanum doped PZT a reduced concentration of oxygen vacancies but increased concentration of lead vacancies results. ${ }^{29}$

Up to now studies on PLZT focused either on compositions well on the rhombohedral side with doping levels up to $10 \%^{34,35}$ or on different doping levels for a single morphotropic composition. ${ }^{36,37}$ For this reason we investigated the structural influence of lanthanum doping for a compositional range across the entire MPB.

\section{EXPERIMENTAL}

$\mathrm{Pb}_{1-(3 / 2) y} \mathrm{La}_{y} \mathrm{Zr}_{1-x} \mathrm{Ti}_{x} \mathrm{O}_{3} \quad$ (PLZT, $\left.100(1-x) / 100 x\right)$ samples were prepared by the mixed-oxide route ${ }^{38}$ with $\mathrm{Zr} / \mathrm{Ti}$ contents across the entire compositional range of the MPB of $56 / 44,55 / 45,54 / 46,53 / 47,52.5 / 47.5,52 / 48$, and 50/50. Donor doping with $1 \mathrm{~mol} \% \mathrm{La}^{3+}$ was performed substituting $\mathrm{Pb}^{2+}$ on the A-site. Powders were pressed uniaxially into cylindrical pellets of $12 \mathrm{~mm}$ in diameter at $17.7 \mathrm{MPa}$, followed by cold isostatic pressing at $400 \mathrm{MPa}$. The pellets were then sintered at $1323 \mathrm{~K}$ for $6 \mathrm{~h}$ with an initial heating rate of $2 \mathrm{~K} / \mathrm{min}$ up to this temperature.

The TEM work was done using a FEI CM20 ST (Super Twin, FEI Eindhoven, Netherlands) at $200 \mathrm{kV}$ with a double tilt sample holder. The samples were prepared from sintered ceramic cylinders $(6 h, 1323 K)$ by successively cutting, grinding, and polishing them to a thickness of around $150 \mu \mathrm{m}$. The ultrasonically cut disks were dimpled from one side to around $20 \mu \mathrm{m}$ and Ar-ion thinned to perforation. To avoid charging in the microscope the samples were coated with a thin carbon layer.

All temperature dependent X-ray measurements were performed at the beamline B2, HASYLAB/DESY, Hamburg, Germany $^{39}$ at an incident wavelength of $\lambda \approx 0.50 \AA(25 \mathrm{keV})$ using a Stoe and Cie capillary furnace for high temperature measurements and a closed cycle capillary cryostat (Cryophysics $^{40}$ for low temperature measurements. Data were recorded by a position-sensitive image-plate detector (OBI, ortsfest auslesbarer Bildplattendetektor) ${ }^{41}$ from $20 \mathrm{~K}$ to well above the paraelectric cubic phase transition in temperature steps of 10 or $20 \mathrm{~K}$. In the temperature interval around a phase transition the steps were smaller.

Additional high resolution X-ray measurements at room temperature were performed at the powder diffraction station of the materials science beamline at the Swiss Light Source (SLS, Villigen, Switzerland) $)^{42}$ at an incident wavelength of $\lambda \approx 0.44 \AA(28 \mathrm{keV})$ using transmission geometry. Data were recorded by a position-sensitive microstrip detector (MYTHEN II). ${ }^{43}$

Neutron powder diffraction measurements were carried out on the Structure Powder Diffractometer (SPODI) at the research reactor FRM-II (Garching b. München, Germany) ${ }^{44}$ at an incident wavelength of $1.548 \AA$. The compositions
PLZT 50/50, 52/48, 52.5/47.5, 53/47, 54/46, 55/45. and $56 / 44$ were measured at room temperature. Low temperature measurements were performed using a closed cycle helium cryostat at $5 \mathrm{~K}$ for the compositions PLZT 50/50, 52/48, $52.5 / 47.5,53 / 47,54 / 46$, and 56/44. Data were collected by a bank of 80 position-sensitive ${ }^{3} \mathrm{He}$ detectors covering a $160^{\circ}$ scattering range.

The full profile Rietveld refinements of the temperature dependent X-ray measurements were carried out using the general structure analysis system (GSAS) ${ }^{45}$ The peak profile shape was described by a Thompson-Cox-Hastings pseudoVoigt function. The background of the DP was fitted by a linear interpolation between selected data points in nonoverlapping regions. The scale factor, lattice parameters, isotropic displacement parameters, sample position shift, and profile shape parameters were varied during the combined fitting of $\mathrm{X}$-ray and neutron diffraction data.

High resolution X-ray and neutron data analysis was performed with the software package FULLPROF. ${ }^{46}$ Microstructural effects are treated within FULLPROF using a pseudoVoigt approximation: both instrumental and sample intrinsic profiles are approximated by an addition of Lorentzian and Gaussian components. The integral breadth is calculated from the Rietveld refinement model of the DP based on the De Keijser formula ${ }^{47}$ after subtraction of instrumental broadening and the implementation of a model for anisotropic peak broadening as developed by Stephens. ${ }^{48}$ This model considers the case of an anisotropic distribution of strain, in which the diffraction peak width increases in proportion to the diffraction order. The instrumental broadening is determined by a Rietveld fit of a high resolution measurement recorded at ambient temperature of the standard reference material $\mathrm{LaB}_{6}$ (SRM 660a, National Institute of Standards, NIST) for X-ray measurements and Si (SRM 640c, National Institute of Standards, NIST) for neutron measurements.

For the refinement of PZT and PLZT a combination of $P 4 \mathrm{~mm}$ and $\mathrm{Cm}$ was used for neutron and X-ray data at room temperature and all temperature dependent X-ray data. For the refinement with low temperature neutron data a combination of $C c$ and $C m$ was used. In a highly correlated system like morphotropic PZT a refinement with more than two phases does not lead to reasonable results. Therefore, the two monoclinic phases were used to investigate a continuous composition dependent phase transition at low temperatures. Due to the expected phase transitions occurring in this temperature and composition range both phases have to be considered as model phases in these refinements. $C c$ accounts for material with superstructure reflections and $\mathrm{Cm}$ accounts for material not transformed to a low temperature phase. $C c$ can be interpreted similar to $\mathrm{Cm}$ according to Fig. 1 and Eq. (1) after a cell transformation.

\section{RESULTS}

We will first concentrate on the TEM and high resolution powder diffraction measurements at room temperature in comparison with previously published data of undoped PZT. ${ }^{13}$ Then, we focus on temperature dependent measurements of PLZT and discuss them with data obtained from 

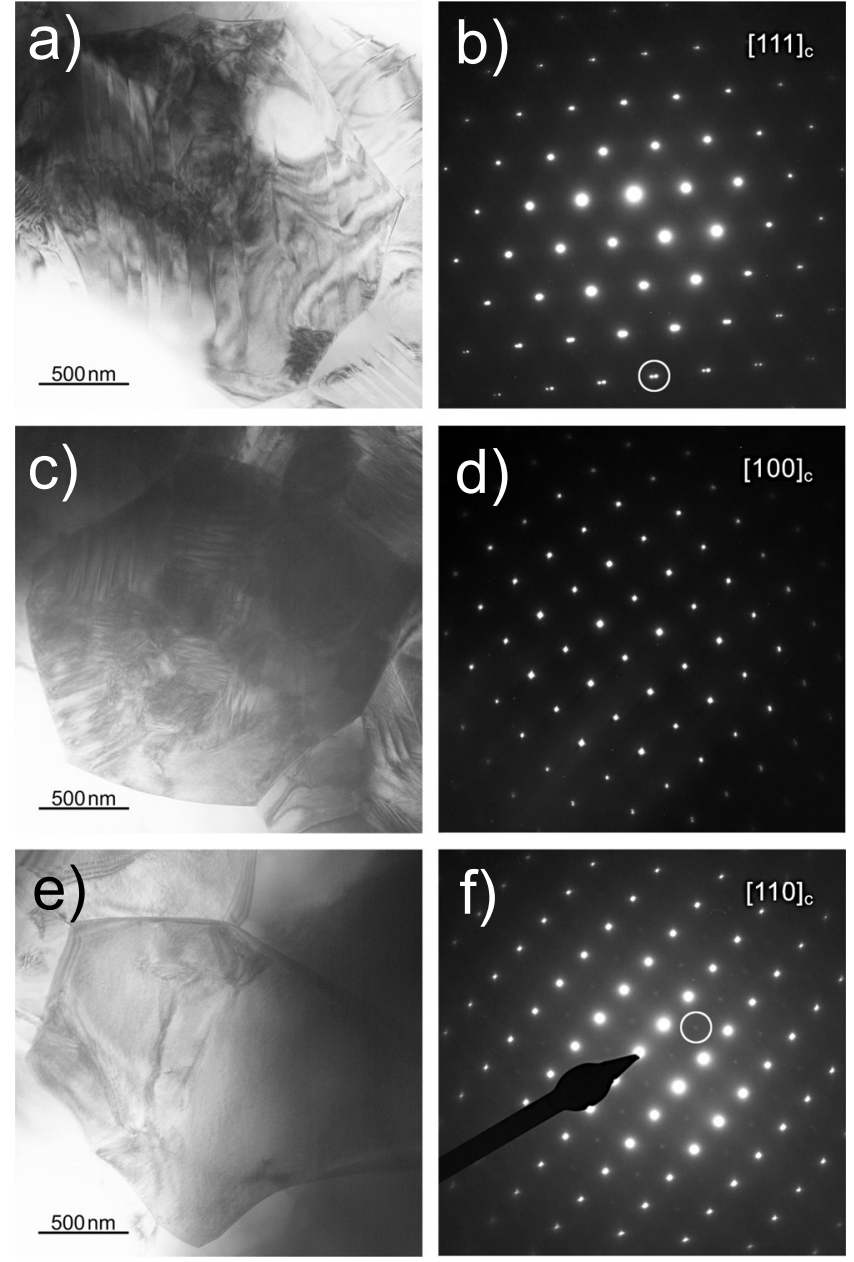

FIG. 2. TEM pictures of PLZT with a Ti content of $47.5 \%$ (a), $46 \%$ (c), and $44 \%$ (e) and corresponding selected area electron DP of PLZT with a Ti content of $47.5 \%$ (b), $46 \%$ (d), and $44 \%$ (f).

PZT. ${ }^{14}$ Finally low temperature neutron powder diffraction measurements complement the observations with X-ray diffraction (XRD).

\section{A. Room temperature}

\section{TEM}

Figure 2 shows the TEM images and the corresponding electron DP for the compositions PLZT 52.5/47.5 [Figs. 2(a) and 2(b)], PLZT 54/46 [Figs. 2(c) and 2(d)], and PLZT 56/44 [Figs 2(e) and 2(f)]. The domain configuration for PLZT $52.5 / 47.5$ is characterized by lamellar domains. Bimodal domain configurations are observed in several grains, comparable to tetragonal undoped PZT. ${ }^{17}$ In Fig. 2(a) on the left side a bimodal configuration is depicted. The small domains are in the range of 20 to $45 \mathrm{~nm}$, while the wider domains are in the range of 50 to $90 \mathrm{~nm}$ and become larger on the right side of the grain. The DP [Fig. 2(b)] clearly shows splitting of higher order reflections [exemplary encircled in Fig. 2(b)], due to the existence of domains. The spots are split along $\langle 110\rangle$ in the $[111]_{c}$-zone axis. On the basis of rhombohedral or tetragonal symmetry, the direction of the splitting is calculated and from the separation of split diffraction points the resulting $c / a$ ratio or rhombohedral angle are obtained. ${ }^{19}$ Derived from the [100]- and [111]-zone axes and in comparison to undoped domain configurations the structure is proposed to be tetragonal. The resulting $c / a$ ratio with around 1.024 is in good agreement with the XRD measurements which yield a $c / a$ ratio of 1.0237 for the same composition.

PLZT 54/46 shows a more complex domain configuration. Unlike in PLZT 52.5/47.5 there are several domain configurations in one grain. The domains are still mainly lamellar but with very small domain sizes. Figure 2(c) represents such a grain with several domain configurations. The corresponding DP [Fig. 2(d)] shows less splitting than for PLZT $52.5 / 47.5$, arguing for a decreased $c / a$ ratio. In the neighboring grain on the bottom right a very fine domain contrast and a fine contrast within domains can be observed. Although there are not as many nanodomains within microdomains as in undoped PZT, the domain width in La-doped material is comparable with the width determined for nanodomains in undoped PZT. ${ }^{17}$

The domain configuration for PLZT 56/44 changes considerably. The domains become wavy and wedge shaped [Fig. 2(e)]. Some grains do not show any domains at all. A common feature for this composition is a grainy contrast [see center of Fig. 2(e) and also in the neighboring grain on top]. A similar contrast is reported for relaxor ferroelectrics (tweed structure) or PZT with higher La content. ${ }^{35}$ In contrast to the other two compositions, PLZT 56/44 shows superstructure reflections of type $1 / 2($ ooo $)$ in the $[110]_{c}$-zone axis, where ' $o$ ' stands for odd miller indices [Fig. 2(f)]. This indicates a crystal structure change with composition to the antiferrodistortive low temperature phase reported as $C c^{5,6}$ or $R 3 c .^{22}$ For undoped PZT, Pandey et al. ${ }^{11}$ reported this phase transition to occur at around $200 \mathrm{~K}$.

\section{High resolution powder diffraction}

For the PLZT compositions around the MPB a combined refinement with high resolution X-ray and neutron data measured at room temperature was carried out. The fits for PLZT 54/46 are depicted in Fig. 3. In the X-ray refinement the pseudocubic $(111)_{c}$ and $(200)_{c}$ reflections are magnified for interpretation. The $(111)_{c}$ reflection pair clearly shows the rhombohedral splitting, whereas in the $(200)_{c}$ reflection pair three separated intensities can be observed. The monoclinic phase proposed by Noheda et al. is set up in such a way that the $(200)_{c}$ reflection is split in a $(002)_{m}$ and a $(220)_{m}$ reflection. ${ }^{1}$ The $(220)_{m}$ reflection cannot be split in this setup resulting in only two reflections for the $(200)_{c}$ reflection. Since $(200)_{c}$ is a singlet in rhombohedral and a doublet in tetragonal symmetry, this is a clear indication for a twophase coexistence.

Four order parameters obtained from the Rietveld refinement are depicted in Fig. 4. The model phase fractions for both undoped PZT ${ }^{13,14}$ and PLZT are plotted in Fig. 4(a). A slight shift to the tetragonal side can be observed for lanthanum doping while the slope is unchanged for both systems. The pseudocubic $c_{m} / a_{p c}$ ratio obtained from the monoclinic cell parameters in Fig. 4(b) is in good agreement with the plot of the tetragonal phase fractions. The decrease in monoclinic phase fraction is governed by an increase in the $c_{m} / a_{p c}$ ratio. The $a_{m} / b_{m}$ ratio displays the distortion of the $\{001\}_{c}$ 


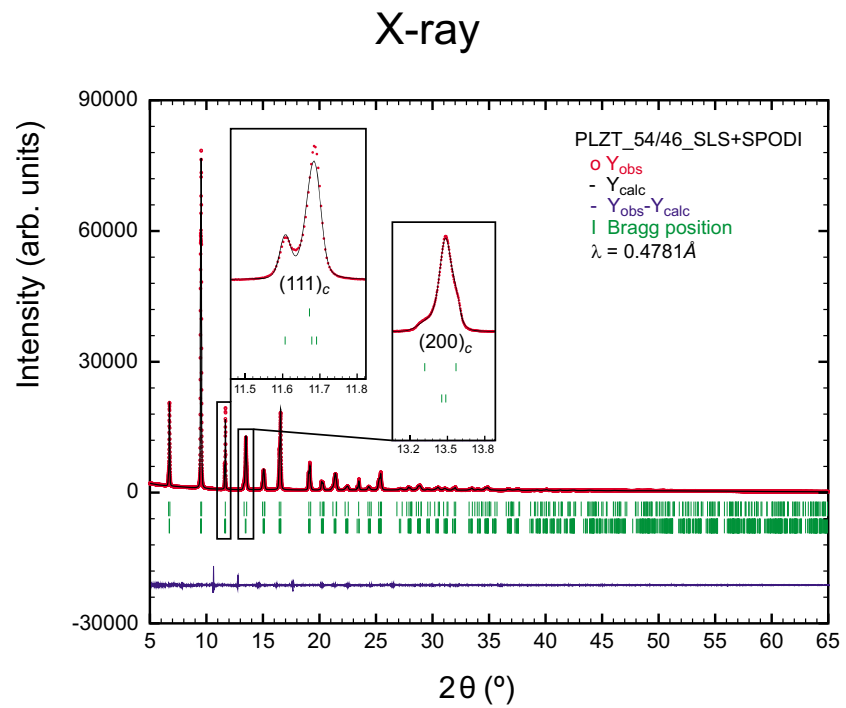

neutron

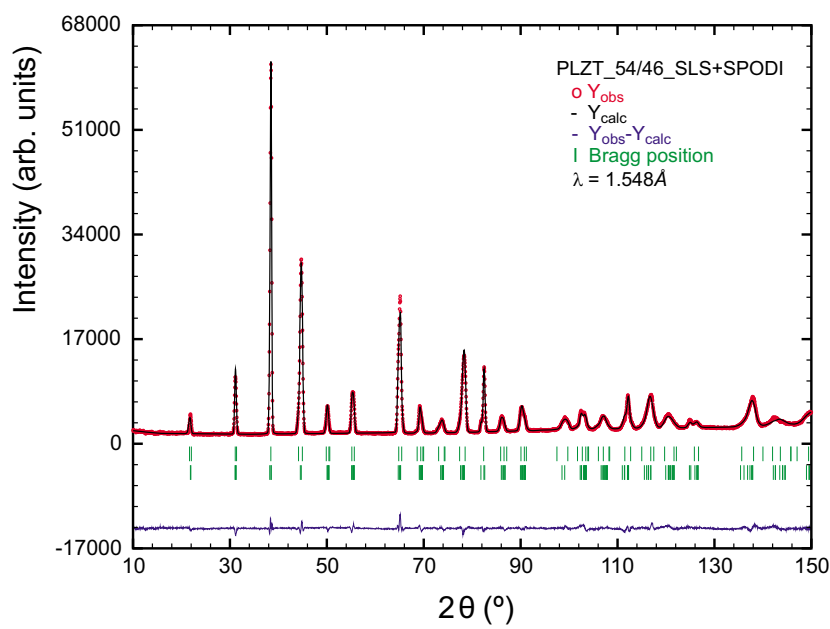

FIG. 3. (Color online) Rietveld refinement fit with high resolution X-ray and neutron diffraction data of PLZT 54/46. The insets show the (111) $)_{c}$ and (200) reflection for interpretation.

plane. The plot in Fig. 4(c) shows an analysis of the lattice parameters of the model phase $\mathrm{Cm}$. For a Ti content above $50 \%$ the pseudorhombohedral angle is calculated as $\alpha_{r}$ $\approx 90^{\circ}$ based on Eq. (1). For low Ti contents $\alpha_{r}$ approaches a common value indicating a systematic change across the MPB [Fig. 4(c)].

Apart from a value change at the MPB, $\beta_{m}$ stays constant on the rhombohedral as well as on the tetragonal side of the phase diagram and well above $90^{\circ}$. Figure 4(d) shows the angle of rotation in the $\{110\}_{c}$ plane away from the $[001]_{c}$ direction. The calculated values of the spontaneous polarization show no significant change across the MPB. With around $40 \mu \mathrm{C} / \mathrm{cm}^{2}$ the values are in the range of undoped morphotropic $\mathrm{PZT}^{23}$

a)

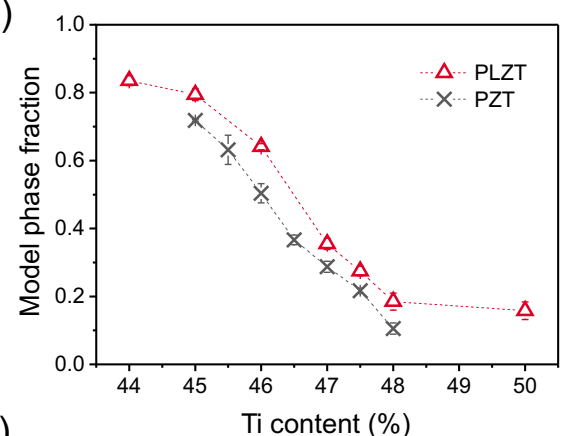

c)

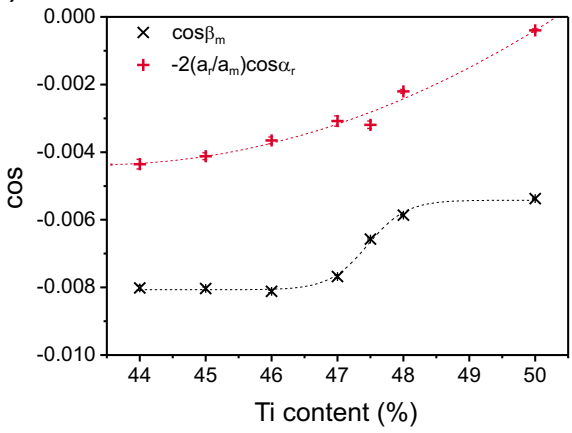

Figures 4(b) and 4(c) together allow a characterization of the model phase $\mathrm{Cm}$. On the tetragonal side $a_{m} \approx b_{m}, \beta_{m}$ $>90^{\circ}$, and $\alpha_{r} \approx 90^{\circ}$, concerning the high phase fraction of $P 4 \mathrm{~mm}$ the structure can be considered as tetragonal. On the rhombohedral side $a_{p c} \approx c_{m}$ and $\alpha_{r}<90^{\circ}<\beta_{m}$ argue for rhombohedral symmetry. But since $-2\left(a_{r} / a_{m}\right) \cos \alpha_{r}$ $\neq \cos \beta_{m}$ the condition for exact rhombohedral symmetry is not fulfilled even for PLZT 56/44. For the morphotropic compositions with a Ti content between $46 \%$ and $48 \%$ a strongly distorted cell with $a_{m} \neq b_{m} \neq c_{m}$ and $\alpha_{r}<90^{\circ}<\beta_{m}$, while $-2\left(a_{r} / a_{m}\right) \cos \alpha_{r} \neq \cos \beta_{m}$ results.

Microstructural analysis was done with the anisotropic peak broadening model from Stephens. ${ }^{48}$ The anisotropic

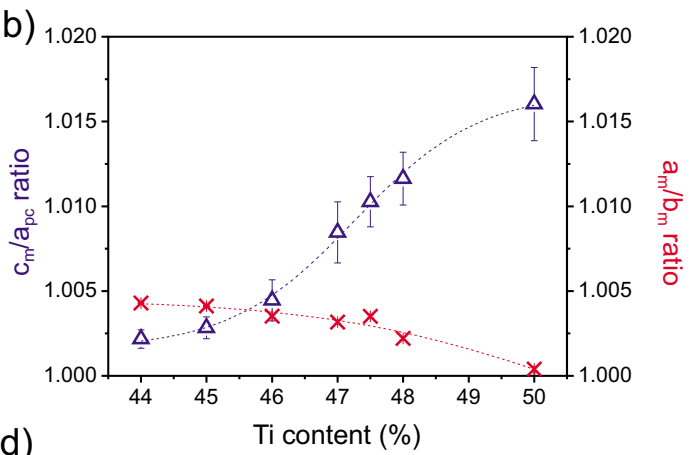

d)

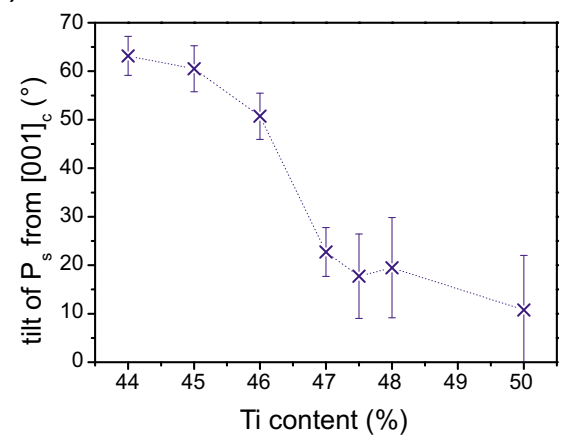

FIG. 4. (Color online) Order parameters obtained from Rietveld refinement with high resolution X-ray and neutron powder diffraction data for interpretation of model phase $C m$ : (a) phase fractions of $C m$ for PZT and PLZT, (b) $a_{m} / b_{m}$ ratio and the pseudocubic $c_{m} / a_{p c}$ ratio, (c) plot of the relation between $\beta_{m}$ and $\alpha_{r}$ as described in Eq. (1), and (d) tilt angle of spontaneous polarization away from [001] $]_{c}$ of PLZT. 

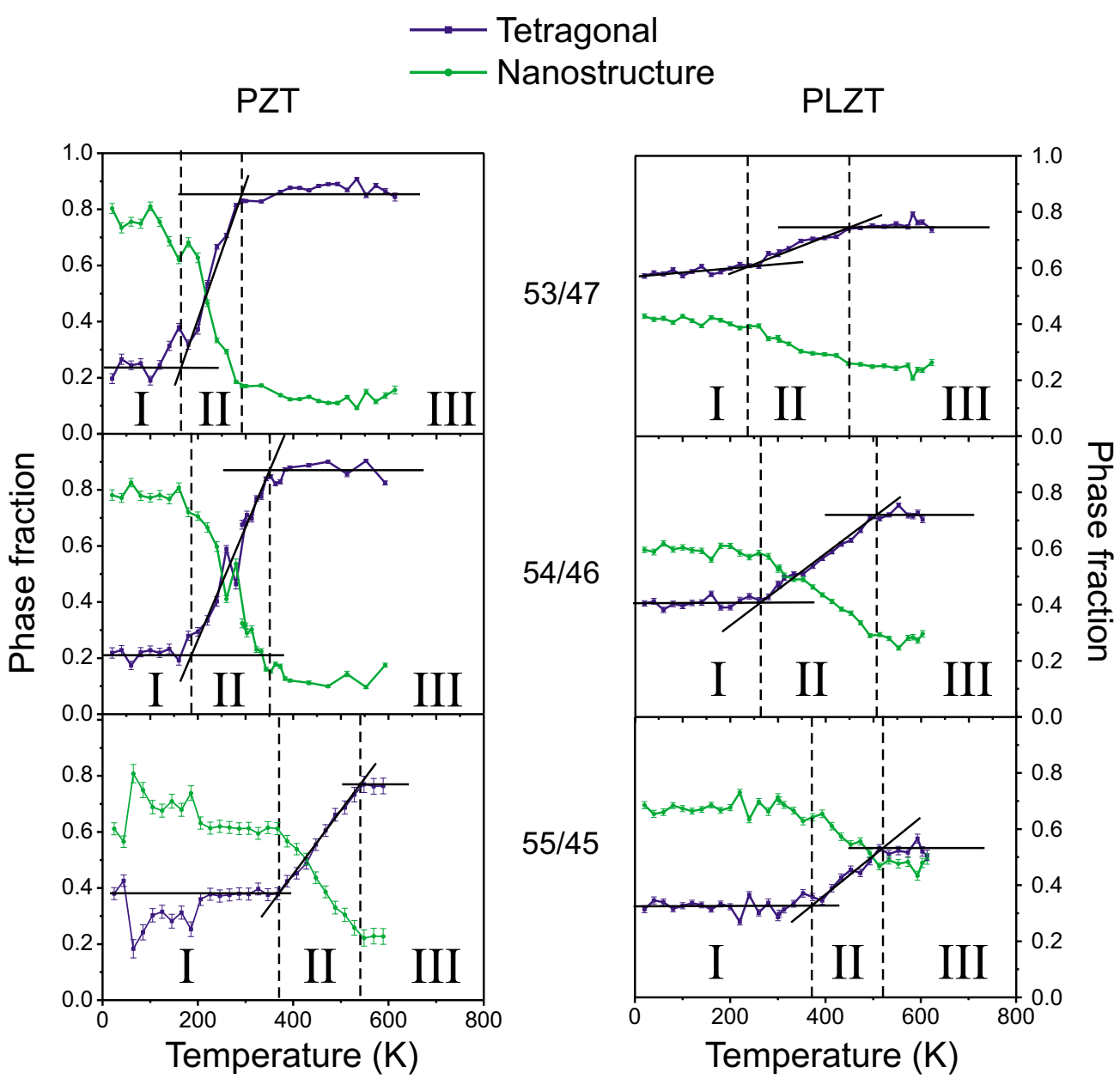

FIG. 5. (Color online) Phase fractions of a two-phase refinement with space groups $P 4 m m$ and $C m$ and graphical estimation of phase transitions of PZT ${ }^{14}$ and PLZT derived from temperature dependent XRD.

strain values calculated with this approach show a direct correlation with the phase fractions of each phase. A decrease in phase fraction correlates with an increase in its strain. In the tetragonal phase highest strains are reached in the polar direction for $(00 l)_{t}$ reflections. In the monoclinic model phase highest strains are reached in the plane of the rotating polarization and especially for $(00 l)_{m}$ and $(h 00)_{m}$ reflections.

\section{B. Temperature dependence}

\section{High temperature}

All high temperature results were obtained from X-ray powder diffraction measurements. The phase fractions for three different compositions depicted in Fig. 5 were derived from a two-phase Rietveld fit with space groups $P 4 \mathrm{~mm}$ and $\mathrm{Cm}$. The formation of the phase fractions as a function of temperature is divided into three ranges

At low temperatures the $\mathrm{Cm}$ phase fraction dominates. This is in agreement with the observations of a low temperature superstructure phase $C c$ already described by Hatch et al. ${ }^{5}$ and Pandey and Ragini ${ }^{6}$ since the superlattice reflections cannot be observed in these measurements.

(II) In an intermediate range around room temperature a changeover of the dominating symmetry takes place, marking the transition between the growth of nanodomains to tetragonal microdomains.

(III) At higher temperatures up to the Curie temperature $\left(T_{c}\right)$ the tetragonal $P 4 \mathrm{~mm}$ symmetry is dominant.

Comparing the development of the phase fractions of $\mathrm{PZT}^{14}$ and PLZT some major differences become apparent. Focusing on the transition between range (II) and (III) on the tetragonal side of the phase diagram, PZT and PLZT samples with a Ti content of at least $46 \%$ show a similar behavior for the high temperature phase below the Curie temperature [range (III), Fig. 5] but PZT shows a large quantity of tetragonal microdomains above the phase transition (II)-(III), while PLZT still exhibits a considerable amount of nanostructures.

For PLZT samples on the rhombohedral side of the phase diagram with a maximum Ti content of $45 \%$ the temperature dependent behavior differs from the behavior of PZT with the same Ti content. The amount of monoclinic phase fraction is significantly higher above the phase transition (II)-(III).

Below the low temperature phase transition (I)-(II) all PZT compositions show similar characteristics which correspond to the antiferrodistortive phase already discussed in the literature ${ }^{5,6}$ with space group $C c$. As no superstructure 


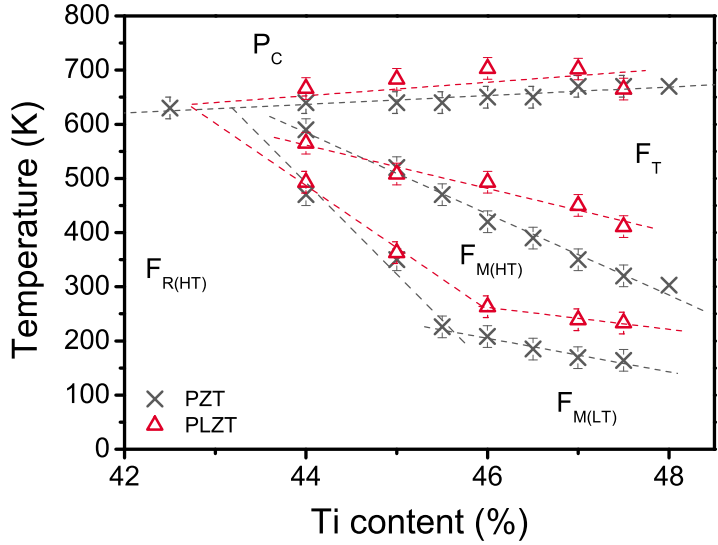

FIG. 6. (Color online) Phase diagram of the MPB derived from temperature dependent XRD.

reflections originating from oxygen octahedral tilting were detected with X-rays, refinement was carried out using $\mathrm{Cm}$ symmetry.

The broad transition range between the low and the high temperature regions [range (II)] shows distinct differences between PZT and PLZT. Especially on the tetragonal side of the phase diagram PZT shows a steeper and, therefore, more conventional transition. On the rhombohedral side the transition is stretched over a wider temperature range and also the amount of material transformed is lower. In PLZT the phase change is generally weak, with a maximum for a Ti content of $46 \%$. This results in a similar behavior of low and high temperature phase for the tetragonal as well as the rhombohedral side of the phase diagram.

The graphs in Fig. 5 show constant values for the phase fractions in range (I) (low temperature phase) as well as for the high temperature phase [range (III)]. The boundaries of these three phase regions are graphically estimated in Fig. 5 with black lines. The intersections of these lines are marked in the phase diagram (Fig. 6) with crosses $X$ for undoped PZT and triangles $\Delta$ for PLZT. Due to the narrow range of composition the shift in the MPB for lanthanum doped PZT is clearly visible. Furthermore, the low temperature phase transition is shifted to higher temperatures and the stability range of nanodomains is extended over a wide composition range. To confirm the increase in the transition temperature, the Curie temperature was additionally estimated by profile fitting of the tetragonal split $(200)_{c}$ and the rhombohedral split $(111)_{c}$ reflections. Both Rietveld refinement and profile fitting yielded a small increase in the Curie temperature for PLZT compared to undoped PZT in the vicinity of the MPB. This is in contrast to observations for doping with higher La content described in the literature. ${ }^{27}$

The three dimensional plot of the temperature and composition dependent evolution of the tetragonal phase fractions from Rietveld refinement, depicted in Fig. 7, shows the influence of lanthanum doping more clearly. In PZT a plateau develops at high temperatures and high Ti content, indicating almost $100 \%$ tetragonal phase fraction. For low temperatures the range of the low temperature superstructure phase reported in the literature, ${ }^{5,6}$ is indicated by constantly low tetragonal phase fractions.

Comparing the 3D contour plot of PZT with the one of PLZT some important differences become obvious. On the tetragonal as well as on the rhombohedral side of the depicted composition range of PLZT only slight variations in phase fractions take place with temperature. Just for compositions in the stability range of nanodomains around $46 \% \mathrm{Ti}$ a considerable shift from rhombohedral to tetragonal symmetry is noticed. Lanthanum doping induces a broadening of the transition range in terms of temperature and composition. The change in phase fractions for the two-phase model is smeared out over a wide temperature range. As described above a two-phase stability can be identified in PLZT but in contrast to PZT the reaction to temperature or compositional variations is damped over a wide temperature range.

\section{Low temperature}

At low temperatures high resolution neutron diffraction was performed at $5 \mathrm{~K}$ for PLZT 50/50, 52/48, 52.5/47.5, $53 / 47,54 / 46$, and 56/44. To determine the transitions between the low temperature phases we refined the neutron diffraction data with a two-phase model of $\mathrm{Cc}$ and $\mathrm{Cm}$, where $\mathrm{Cm}$ accounts for the phase fraction without octahedral tilting. An exemplary Rietveld refinement of PLZT 54/46 is
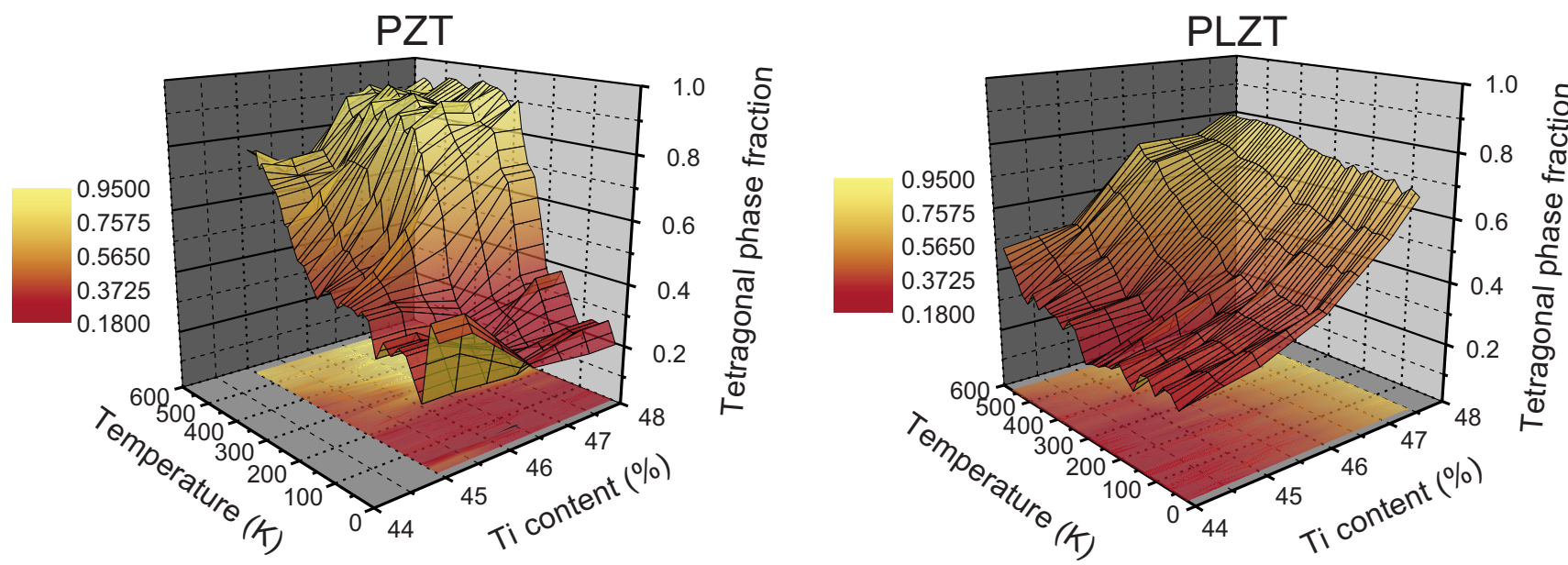

FIG. 7. (Color online) 3D contour plot of the tetragonal phase fractions of the two-phase refinement vs temperature and Ti content of PZT ${ }^{14}$ and PLZT. 
a)

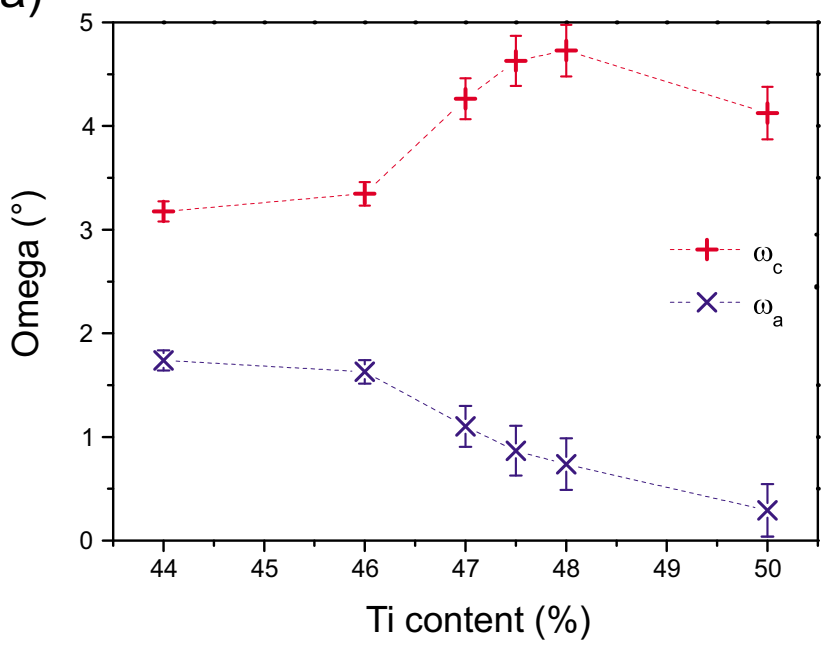

b)

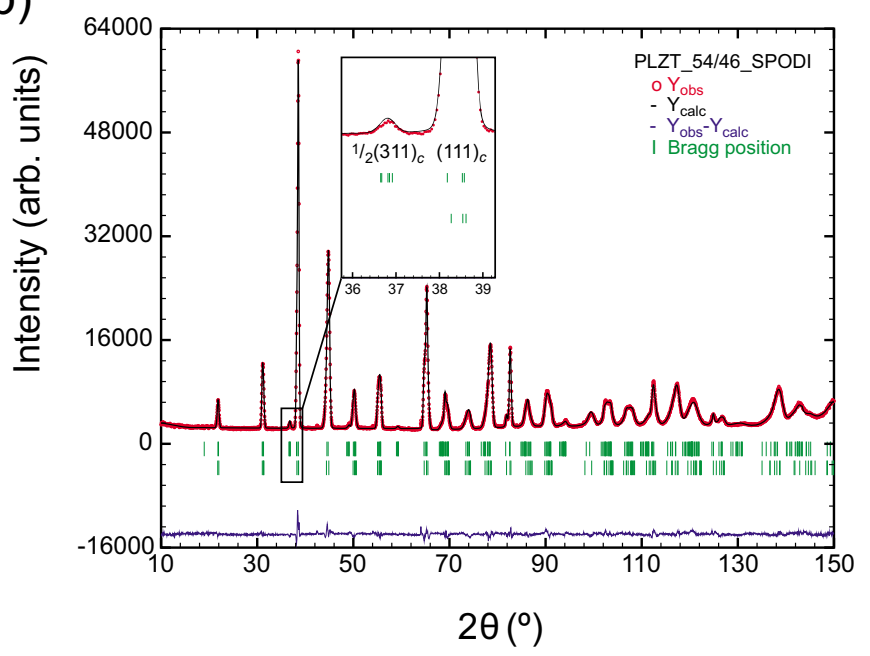

FIG. 8. (Color online) (a) Octahedral tilting angles of $C c$ and (b) Rietveld refinement fit of PLZT 54/46 with high resolution neutron data at 5 K. The inset shows the $1 / 2(311)_{c}$ superlattice reflection.

shown in Fig. 3(b). After a cell transformation the low temperature $C c$ phase can be interpreted similar to $\mathrm{Cm}$ in the refinements above room temperature.

The low temperature phase is characterized by superstructure reflections due to oxygen octahedral tilting [inset Fig. 8(b)]. Therefore, only neutron diffraction measurements were considered for a detailed structural analysis. The two octahedral tilting angles $\omega_{a}$ and $\omega_{c}$ of the $a^{-} a^{-} c^{-}$tilt system in $C c$ are depicted in Fig. 8(a). From the results three main regions can be identified. For the two samples with Ti contents up to $46 \%$ on the rhombohedral side the two angles are converging in the direction of lower Ti content. For $\omega_{a}=\omega_{c}$ the $a^{-} a^{-} a^{-}$tilt system with space group $R 3 c^{3}$ would be appropriate. For higher $\mathrm{Ti}$ contents of around $50 \% \omega_{a}$ approaches zero resulting in an $a^{0} a^{0} c^{-}$tilt system which can be described with the space group $14 \mathrm{~cm} .^{7}$ In the intermediate composition range $\omega_{c}$ reaches a maximum while $\omega_{a}$ decreases constantly.

For the measurements at $5 K$ the bordering space groups $R 3 \mathrm{c}$ and $I 4 \mathrm{~cm}$ have been tested with similar parameter sets in a two-phase fit with $\mathrm{Cm}$. On the tetragonal side it was not possible to reach a similar agreement of calculated and observed data as for the two-phase fit with $C c$. Table I shows the agreement factors of the refinements. On the rhombohedral side both refinements show comparable agreement factors with still a better fit for $\mathrm{Cc}$ and $\mathrm{Cm}$.

When comparing $\mathrm{Cm}$ from room temperature refinements with $C c$ from low temperature refinements both phase fractions show a similar dependence on Ti content. A continuous decrease in the $C c$ phase fraction from around $77 \%$

TABLE I. Agreement factors of the low temperature refinement.

\begin{tabular}{lccr}
\hline \hline & $R_{p}$ & $R_{w p}$ & \multicolumn{1}{c}{$\chi^{2}$} \\
\hline PLZT 50/50 Cm+Cc & 3.73 & 4.88 & 7.30 \\
PLZT 50/50 Cm+I4cm & 4.38 & 6.00 & 10.90 \\
PLZT 56/44 Cm+Cc & 2.61 & 3.34 & 5.68 \\
PLZT 56/44 Cm+R3c & 2.77 & 3.51 & 6.28 \\
\hline \hline
\end{tabular}

to around $22 \%$ for an increasing Ti content from $44 \%$ to $50 \%$ indicates a systematic change with composition. In the same way the pseudocubic $c_{p c} / a_{p c}$ ratio increases. The structural parameters of the $\mathrm{Cm}$ phase exhibit a similar behavior as for room temperature. The $c_{m} / a_{p c}$ ratio increases with increasing $\mathrm{Ti}$ content, while the monoclinic and the pseudorhombohedral angle decrease. The spontaneous polarization shows similar values as for room temperature with no significant variation dependent on composition. For $5 K$ the strain for both phases follows the same dependence on phase fractions as described above for room temperature.

\section{DISCUSSION}

The most obvious modification induced by lanthanum doping is highlighted by the temperature and composition dependent evolution of the phase fractions (Fig. 5). The behavior of the individual phase fractions in PZT and PLZT originates from the different microstructures of the two materials. While in tetragonal PZT microdomains of the size of around hundred nanometers are subdivided by nanodomains of the size of a few nanometers, ${ }^{12,13}$ tetragonal PLZT does not show clear nanodomains within domains. All dimensions in the PLZT domain structure are of a similar magnitude as the nanodomains in PZT (Fig. 2).

In tetragonal PLZT the model phase $\mathrm{Cm}$ is close to the tetragonal parent phase. The $\{001\}_{c}$ plane shows no distortion for PLZT 50/50 and the direction of the spontaneous polarization is close to $[001]_{c}[$ Fig. $4(\mathrm{~d})]$ but the angle $\beta_{m}$ is still well above $90^{\circ}$. Due to the high phase fraction of $P 4 \mathrm{~mm}$ the structure can be assumed as tetragonal with small domain structures [Fig. 4(c)].

On the rhombohedral side of the phase diagram PLZT only shows small amounts of nanodomains, but a grainy contrast [Fig. 2(e)], which can be explained by local polarization differences. The interpretation of the model phase $\mathrm{Cm}$ with the ratios of the cell parameters in Fig. 4(b) shows, that in PLZT 56/44 the cell of $\mathrm{Cm}$ is compressed along $b_{m}$, i.e., perpendicular to the polarization rotation. The lattice param- 
eters $a_{m}$ and $c_{m}$ agree with the conditions necessary for an interpretation as rhombohedral microdomains as described in Fig. 1. On the rhombohedral side $-2\left(a_{r} / a_{m}\right) \cos \alpha_{r}$ asymptotically approaches a common value well above $\cos \beta_{m}$. Hence the structure in this composition range can only be modeled as monoclinic. For lower Ti contents the structure might converge to larger domains resolving their internal symmetry again in diffraction. Yokota et al. ${ }^{22}$ suggested that even far on the rhombohedral side PZT still consists of two phases, namely, rhombohedral and monoclinic. Since this study focuses on the phase transitions across the MPB the introduction of a third coexisting phase would lead to problems of underdetermination. Additionally the compositional range would need to be extended further to the rhombohedral side to investigate this range. Therefore, based on results from TEM and powder diffraction, we interpret this region as highly disordered microdomains.

The most pronounced change in phase fractions is found at a $\mathrm{Ti}$ content of $46 \%$, where the transition region is stretched over a wide temperature range and the high resolution measurements at room temperature clearly show a phase coexistence of tetragonal microdomains and nanodomains, because of the pronounced splitting of the (111) as well as the $(200)_{c}$ reflection (Fig. 3). In the applied twophase fit the model phase $\mathrm{Cm}$ can neither be interpreted as rhombohedral nor as tetragonal microdomains. Regarding the TEM observations with large amounts of nanodomains [Fig. 2(c)] and the order parameters in Fig. 4 the theory of Glazer et $a{ }^{9}{ }^{9}$ could be considered: at the MPB the local ordering of $\mathrm{Pb}$ displacements is extended to long range order of a size sufficient that diffraction techniques see a distinct phase of monoclinic symmetry, which was reported by Schierholz et al. for undoped PZT. ${ }^{18,19}$

The domain structures in PLZT 54/46 have a size of several nanometers and the characteristics of a tetragonal microstructure but compared to $\mathrm{PZT}^{13}$ no superordinated microdomain structures are visible. The local $\mathrm{Pb}$ displacements could extend to long range order, while lanthanum doping suppresses the formation of microdomains. However, due to coherence effects $^{20}$ of the highly disordered microstructure [Fig. 2(c)], the reflections of the model phase are severely influenced, hindering a clear interpretation.

In Fig. 3 the phase coexistence of nanostructures and tetragonal microdomains is highlighted by the triple split $(200)_{c}$ reflection. The order parameters in Fig. 4 show that the model phase is far away from tetragonal or rhombohedral symmetry. The $c_{m} / a_{p c}$ ratio is well above 1 and the direction of the spontaneous polarization lies between the values expected for tetragonal $\left(0^{\circ}\right)$ and rhombohedral $\left(54.7^{\circ}\right)$, while the condition of Eq. (1) is not fulfilled. In the other regions $\mathrm{Cm}$ is either pseudotetragonal or pseudorhombohedral.

On the tetragonal side $\mathrm{Cm}$ is only present as a residual of the refinement $(\sim 10 \%)$ so the structure is purely tetragonal as seen averaged by X-ray and neutron diffraction. On the rhombohedral side the model phase exhibits pseudorhombohedral characteristics. While crossing the MPB, dependent on $x$ the amount of microdomains is reduced and transformed to nanodomains. Due to a large amount of microdomains on the tetragonal side the model phase is forced to pseudotet- ragonal symmetry. In the two-phase region the model phase exhibits long range order and displays the monoclinic distortion. Progressing to the rhombohedral side the structure comes back to short range order and is averaged to pseudorhombohedral symmetry. This way a continuous composition dependent transition from tetragonal to rhombohedral is enabled through nanodomains.

This is confirmed by the results from microstructural analysis. Highest strain is observed along the directions of the spontaneous polarization. Thus largest deviations of $d$-values are found along the polar axes. For lamellar domains this indicates that the polar axes are oriented along the width of the domains. The best explanation for this configuration are $90^{\circ}$ domains published for $\mathrm{BaTiO}_{3}{ }^{49}$ and $\mathrm{PZT}^{12}$ instead of $180^{\circ}$ domains. This is in good agreement with the results from TEM, since a reflection splitting can only be observed for $90^{\circ}$ domain configurations [Figs. 2(a) and 2(c)].

The combined phase diagram of PZT and PLZT (Fig. 6) clarifies the influence of lanthanum doping. At room temperature the stability range of the nanodomains is extended, shifting the MPB to the tetragonal side. Regarding the whole temperature range the MPB of PLZT is slightly tilted in comparison with the MPB of $\mathrm{PZT}^{14}$ resulting in a broadening of the compositional range influenced by a morphotropic transition. This tilting also involves an elevation of the transition temperature between range (I) and (II). The area determined by graphical estimation of the phase fractions (Fig. 5) marks the two phase region between the tetragonal and the rhombohedral side and the stability range of nanodomains.

The graphical visualization of the composition and temperature dependent evolution of the phase fractions (Fig. 7) draws a more detailed picture of the corresponding region. While the evolution of the tetragonal phase fraction in PZT renders very well the $\mathrm{MPB},{ }^{14}$ the corresponding graph for PLZT depicts a significantly broadened transition in terms of temperature and composition. The $\mathrm{La}^{3+}$ ions interrupt the ordering of the A-site displacements along $[100]_{c},[110]_{c}$, and $[111]_{c}$, resulting in smaller domain structures and favoring a continuous structural transition of the model phase from pseudotetragonal to pseudorhombohedral. The decrease in oxygen vacancies $V_{\mathrm{O}}^{\circ}$ and increase in lead vacancies $V_{\mathrm{Pb}}^{\prime \prime}$ as shown by Eichel and Smyth ${ }^{29}$ due to lanthanum doping, affect a relaxation of the A-site displacements and lead to smaller domain sizes as seen in PLZT 54/46 [Fig. 2(c)] compared to PZT 54/46, ${ }^{13}$ where the effect reaches its maximum.

Compositional changes and a variation in temperature result in weak alterations of the phase configuration, which means that PLZT responds less sensitive to a change in the $\mathrm{Zr}$ /Ti ratio than PZT. Thus the domain configuration is less affected by local variations from the average $\mathrm{Zr} / \mathrm{Ti}$ ratio or in temperature. Especially for the rhombohedral compositions no abrupt microstructural changes occur in narrow temperature intervals during cooling down from above $T_{c}$ (Fig. 5). Thus no long range order of the size of nanodomains precipitates.

The formation of the polarization at the MPB leads to ordering on the local scale resulting in nanodomains without superordinated microdomain structures. In the paraelectric cubic phase six equivalent $[100]_{c}$ directions may transform 
to the tetragonal polarization direction, while the transformation to rhombohedral symmetry offers eight equivalent $[111]_{c}$ directions. Therefore, the same ordering processes apply as for tetragonal compositions but for the formation of polar domains two additional degrees of freedom are accessible resulting in even smaller domain sizes. In the rhombohedral composition under study with $44 \%$ Ti these domains are minimized to the resolution limit of TEM, resulting in the corresponding DP.

Low temperature neutron diffraction results point out, that similar to room temperature a smooth composition dependent turnover from tetragonal to rhombohedral symmetry takes place. This is confirmed by an additional order parameter, the octahedral tilting. On the rhombohedral side $\omega_{a}$ and $\omega_{c}$ are approaching and might even converge for lower Ti contents resulting in the rhombohedral superstructure phase $R 3 c$ with octahedral tilting system $a^{-} a^{-} a^{-}$. Since the agreement factors at a Ti content of $44 \%$ are comparable for the rhombohedral and the monoclinic refinement the phase transition might occur around this composition. Further investigations with an extended composition range are needed to confirm this transition.

On the tetragonal side the octahedral tilting decreases for increasing Ti content with $\omega_{a}$ disappearing for a Ti content of $50 \%$. The interpretation of the lattice and the atomic parameters together with the octahedral tilting indicate a change in the tilting system to $a^{0} a^{0} c^{-}$governed by tetragonal symmetry $I 4 \mathrm{~cm}$. The agreement factors show that a refinement with $I 4 \mathrm{~cm}$ results in less accordance. Together with the decreasing phase fraction of $C c$ the disappearance of the superstructure phase is confirmed for higher Ti contents.

For the compositions at the MPB $\omega_{a}$ and $\omega_{c}$ are clearly separated and well above zero, indicating monoclinic structure according to observations at room temperature, which could be described with an $a^{-} a^{-} c^{-}$tilt system of the space group $C c$. The high strain values especially along the polar axes are similar to room temperature results and indicate an increase in nanodomain content in this region.

Due to the reduced amount of oxygen vacancies $V_{\mathrm{O}}^{*}$ the composition dependent behavior shows the same behavior as for room temperature. The oxygen matrix is significantly relaxed, allowing a continuous transition from rhombohedral to tetragonal symmetry while crossing the MPB. The maximum of $\omega_{c}$ at a Ti level of around $48 \%$ indicates an increase in long range order with significant domain sizes for monoclinic symmetry.

With these results we propose the phase diagram for morphotropic compositions of PZT doped with $1 \%$ lanthanum depicted in Fig. 9. The phase diagram cannot be setup in a classical way separating crystallographic phases from each other. The results from Rietveld refinement in Fig. 5 clearly indicate regions with majority phases but on both sides of the transition region we observe a coexistence of two phases. Therefore, the interpretation of the phases plays an important role. We interpret the model phase $\mathrm{Cm}$ dependent on the lattice parameters resulting in a continuous transition from nanodomains to pseudorhombohedral microdomains. The depiction of the stability range of nanodomains is smeared out in both compositional directions, indicated by

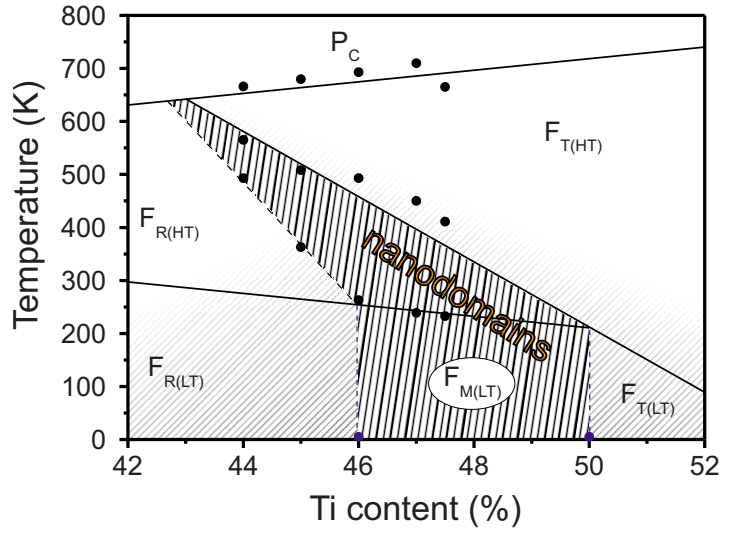

FIG. 9. (Color online) Phase diagram for morphotropic PLZT derived from neutron and XRD.

the shaded area in Fig. 9. The refined structure in the stability range is averaged to clear monoclinic symmetry but the internal structure does not necessarily need to be monoclinic. Schierholz et al. ${ }^{18,19}$ found monoclinic symmetry in single domains in PZT but based on the methods applied in this study we are not able to prove the internal structure of the nanodomains. For low temperature measurements the octahedral tilting angle indicates the phase transitions and shows the same continuous transition from pseudorhombohedral to pseudotetragonal symmetry as the model phase for room temperature. This is indicated by the two vertical lines at $46 \%$ and $50 \%$ Ti content.

\section{CONCLUSION}

The results of the combined refinement with high resolution synchrotron and neutron data together with the observations in TEM provide new insight into the structure of PLZT. At room temperature the symmetry can be described as purely tetragonal for compositions with more than 50\% Ti. With decreasing Ti content the structure of the nanodomains approaches rhombohedral symmetry without reaching it, at least in the composition range under study. The $c_{m} / a_{p c}$ ratio and $\alpha_{r}$ asymptotically approximate values well away from the ones expected for rhombohedral symmetry. At the MPB the nanodomains exhibit increased domain sizes and are the dominant configuration.

\section{ACKNOWLEDGMENTS}

The research leading to these results has received funding from the European Community's Seventh Framework Programme (FP7/2007-2013) under Grant Agreement No. 226716. The authors appreciate the financial support of the German Research Foundation (DFG) through the Sonderforschungsbereich 595 'Electric fatigue in functional materials' and the German Crystallographic Society (DGK).

${ }^{1}$ B. Noheda, D. E. Cox, G. Shirane, J. A. Gonzalo, L. E. Cross, and S.-E. Park, Appl. Phys. Lett. 74, 2059 (1999).

${ }^{2}$ B. Jaffe, W. R. Cook, and H. Jaffe, Piezoelectric Ceramics (Academic, London, 1971).

${ }^{3}$ C. Michel, J.-M. Moreau, G. D. Achenbach, R. Gerson, and W. J. James, Solid State Commun. 7, 865 (1969).

${ }^{4}$ A. M. Glazer, Acta Crystallogr., Sect. B: Struct. Crystallogr. Cryst. Chem. 
B28, 3384 (1972).

${ }^{5}$ D. M. Hatch, H. T. Stokes, R. Ranjan, Ragini, S. K. Mishra, D. Pandey, and B. J. Kennedy, Phys. Rev. B 65, 212101 (2002).

${ }^{6}$ D. Pandey and Ragini, Z. Kristallogr. 218, 1 (2003).

${ }^{7}$ I. A. Kornev, L. Bellaiche, P.-E. Janolin, B. Dkhil, and E. Suard, Phys. Rev. Lett. 97, 157601 (2006).

${ }^{8}$ D. L. Corker, A. M. Glazer, R. W. Whatmore, A. Stallard, and F. Fauth, J. Phys.: Condens. Matter 10, 6251 (1998).

${ }^{9}$ A. M. Glazer, P. A. Thomas, K. Z. Baba-Kishi, G. K. H. Pang, and C. W. Tai, Phys. Rev. B 70, 184123 (2004).

${ }^{10}$ B. Noheda, J. A. Gonzalo, L. E. Cross, R. Guo, S.-E. Park, D. E. Cox, and G. Shirane, Phys. Rev. B 61, 8687 (2000)

${ }^{11}$ D. Pandey, A. K. Singh, and S. Baik, Acta Crystallogr., Sect. A: Found. Crystallogr. A64, 192 (2008).

${ }^{12}$ L. A. Schmitt, Transmissionselektronenmikroskopische Untersuchung der Domänenkonfiguration in $\mathrm{Pb}\left(\mathrm{Zr}_{1-x} \mathrm{Ti}_{x}\right) \mathrm{O}_{3}$-Keramiken im Bereich der morphotropen Phasengrenze (Shaker Verlag $\mathrm{GmbH}$, Aachen, 2008).

${ }^{13}$ K. A. Schönau, L. A. Schmitt, M. Knapp, H. Fuess, R.-A. Eichel, H. Kungl, and M. J. Hoffmann, Phys. Rev. B 75, 184117 (2007).

${ }^{14}$ K. A. Schoenau, In situ Synchrotron Diffraction of Lead-ZirconateTitanate at its Morphotropic Phase Boundary (Shaker Verlag GmbH, Aachen, 2008)

${ }^{15}$ D. I. Woodward, J. Knudsen, and I. M. Reaney, Phys. Rev. B 72, 104110 (2005).

${ }^{16}$ H. Wang, J. Zhu, N. Lu, A. A. Bokov, Z.-G. Ye, and X. W. Zhang, Appl. Phys. Lett. 89, 042908 (2006)

${ }^{17}$ L. A. Schmitt, K. A. Schoenau, R. Theissmann, H. Fuess, H. Kungl, and M. J. Hoffmann, J. Appl. Phys. 101, 074107 (2007).

${ }^{18}$ R. Schierholz, H. Fuess, K. Tsuda, Y. Ogata, and M. Terauchi, Phys. Rev. B 78, 024118 (2008).

${ }^{19} \mathrm{R}$. Schierholz, Konvergente Elektronenbeugung an $\mathrm{PbZr}_{1-x} \mathrm{Ti}_{x} \mathrm{O}_{3}$-Keramiken, Ph.D. thesis, TU Darmstadt, 2010.

${ }^{20}$ H. Boysen, J. Phys.: Condens. Matter 19, 275206 (2007).

${ }^{21}$ Y. U. Wang, Phys. Rev. B 76, 024108 (2007).

${ }^{22}$ H. Yokota, N. Zhang, A. E. Taylor, P. Thomas, and A. M. Glazer, Phys Rev. B 80, 104109 (2009).

${ }^{23}$ J. Frantti, S. Ivanov, S. Eriksson, H. Rundloef, V. Lantto, J. Lappalainen, and M. Kakihana, Phys. Rev. B 66, 064108 (2002).

${ }^{24}$ X. L. Zhang, Z. X. Chen, L. E. Cross, and W. A. Schulze, J. Mater. Sci. 18, 968 (1983)

${ }^{25}$ F. Kulcsar, J. Am. Ceram. Soc. 42, 343 (1959).

${ }^{26}$ X. Dai, Z. Xu, and D. Viehland, J. Am. Ceram. Soc. 79, 1957 (1996).
${ }^{27}$ G. H. Haertling and C. E. Land, J. Am. Ceram. Soc. 54, 1 (1971).

${ }^{28}$ R. Atkin, R. L. Holman, and R. M. Fulrath, J. Am. Ceram. Soc. 54, 113 (1971).

${ }^{29}$ R.-A. Eichel and D. M. Smyth, Acta Mater. (submitted).

${ }^{30}$ C. Miclea, C. Tanasoiu, C. F. Miclea, L. Amarande, A. Gheorghiu, and F. Sima, J. Eur. Ceram. Soc. 25, 2397 (2005).

${ }^{31}$ T. Weston, A. Webster, and V. McNamara, J. Am. Ceram. Soc. 52, 253 (1969).

${ }^{32}$ C. Huang, B. Chen, and L. Wu, Solid State Commun. 130, 19 (2004).

${ }^{33}$ S. Majumder, B. Roy, R. Katiyar, and S. Krupanidhi, J. Appl. Phys. 90, 2975 (2001).

${ }^{34}$ A. Peláiz-Barranco, J. Guerra, O. García-Zaldívar, F. Calderón-Piñar, M. Mendozac, D. Hall, and E. Araújo, Solid State Commun. 149, 1308 (2009).

${ }^{35}$ X. Dai, Z. Xu, J.-F. Li, and D. Viehland, J. Mater. Res. 11, 618 (1996).

${ }^{36}$ R. Withers, Y. Liu, and T. Welberry, J. Solid State Chem. 182, 348 (2009).

${ }^{37}$ K. P. Rema, A. S. Divya, and V. Kumar, J. Phys. D: Appl. Phys. 42, 075420 (2009).

${ }^{38}$ M. Hammer and M. J. Hoffmann, J. Am. Ceram. Soc. 81, 3277 (1998).

${ }^{39}$ M. Knapp, C. Baehtz, H. Ehrenberg, and H. Fuess, J. Synchrotron Radiat. 11, 328 (2004)

${ }^{40}$ J. Ihringer and A. Küster, J. Appl. Crystallogr. 26, 135 (1993).

${ }^{41}$ M. Knapp, V. Joco, C. Baehtz, H. H. Brecht, A. Berghaeuser, H. Ehrenberg, H. von Seggern, and H. Fuess, Nucl. Instrum. Methods Phys. Res. A 521, 565 (2004).

${ }^{42}$ B. Patterson, R. Abela, H. Auderset, Q. Chen, F. Fauth, F. Gozzo, G. Ingold, H. Kuehne, M. Lange, D. Maden, D. Meister, P. Pattison, T. Schmidt, B. Schmitt, C. Schulze-Briese, M. Shi, M. Stampanoni, and P. Willmott, Nucl. Instrum. Methods Phys. Res. A 540, 42 (2005).

${ }^{43}$ B. Schmitt, C. Broennimann, E. F. Eikenberry, F. Gozzo, C. Hoermann, R. Horisberger, and B. Patterson, Nucl. Instrum. Methods Phys. Res. A 501, 267 (2003).

${ }^{44}$ M. Hoelzel, A. Senyshyn, R. Gilles, H. Boysen, and H. Fuess, Neutron News 18, 23 (2007).

${ }^{45}$ B. H. Toby, J. Appl. Cryst. 34, 210 (2001).

${ }^{46}$ T. Roisnel and J. Rodriguez-Carvajal, Mater. Sci. Forum 118, 378 (2001).

${ }^{47}$ T. H. de Keijser, J. I. Langford, E. J. Mittemeijer, and A. B. P. Vogels, J. Appl. Crystallogr. 15, 308 (1982).

${ }^{48}$ P. W. Stephens, J. Appl. Crystallogr. 32, 281 (1999).

${ }^{49}$ Y. H. Hu, H. M. Chan, Z. X. Wen, and M. P. Harmer, J. Am. Ceram. Soc. 69, 594 (1986). 